\title{
Enterite necrótica aviária
}

\author{
Avian necrotic enteritis \\ João Rodrigo Gil de los Santos ${ }^{\mathrm{I}}$ Fabricio Rochedo Conceição ${ }^{I I}$ \\ Carlos Gil-Turnes
}

\section{- REVISÃo BIBLIOGRÁFICA -}

\section{RESUMO}

A Enterite Necrótica Aviária (ENA) é uma enterotoxemia aguda que aparece subitamente e provoca morte rápida, afetando principalmente animais jovens. Embora seu impacto negativo na produção, devido ao aumento da conversão alimentar e da condenação de carcaças seja já conhecido, questões relacionadas à etiologia, à patogenia $e$ ao controle desta importante enfermidade necessitam de maiores esclarecimentos. Nos últimos anos, o controle da ENA baseou-se na aplicação de antibióticos na ração animal, prática banida pelo mercado consumidor, que exigiu o desenvolvimento de novas estratégias de controle. Esta revisão aborda informações sobre a etiologia, a epizootiologia, a patogenia, o diagnóstico e o controle da doença, em especial a utilização de probióticos e vacinas como alternativas de controle da ENA.

Palavras-chave: Enterite Necrótica Aviária, Clostridium perfringens $A$, probióticos, vacinas.

\section{ABSTRACT}

Avian Necrotic Enteritis is an acute enterotoxaemia that appears suddenly producing rapid deaths, affecting mainly young animals. Although its negative impact in poultry production is already known, factors related to etiology, pathogenesis and control of this important disease need better clarifications. For a long time its control was based on the use of antibiotics in poultry feed, whose the use was banned by several consumer markets, requiring the development of new control strategies. Informations on the etiology, epizootiology, pathogenesis, diagnosis and control are reviewed, emphasizing the role of probiotics and vaccines as control alternatives.
Key words: Avian Necrotic Enteritis, Clostridium perfringens A, probiotics, vaccines.

\section{INTRODUÇÃO}

No primeiro trimestre de 2007, a carne de frango ocupou o primeiro lugar na produção animal no Brasil. Nesse período, foi abatido 1 bilhão de frangos, representando mais de 2 milhões de toneladas de carcaças, sendo comercializadas no mercado externo 697,2 mil toneladas, com um aumento de 14,3\% em relação ao mesmo período de 2006 (IBGE, 2007).

Parte dos altos índices de produtividade do setor avícola foi obtida com a utilização de antibióticos na ração animal. Porém, o marcado incremento da resistência bacteriana, incluindo o Clostridium perfringens (JOHANSSON et al., 2004; MARTEL et al., 2004), à grande maioria dos antibióticos foi relacionado a seu uso indiscriminado como promotores de crescimento. Além disso, $\boldsymbol{C}$. perfringens oriundo de frangos de corte também pode ser transmitido a humanos por meio da cadeia alimentar (VAN IMMERSEEL et al., 2004), como foi comprovado por SINGH et al. (2005), que isolaram a bactéria de 70,4\% de amostras de carne de frango obtidas em lojas de varejo na Índia. Esses fatos, entre outros, levaram a

\footnotetext{
'Programa de Pós-graduação em Biotecnologia Agrícola, Centro de Biotecnologia, Universidade Federal de Pelotas (UFPel), Campus Universitário, s/n, 96010-900, Pelotas, RS, Brasil. E-mail: gildelossantos@uol.com.br. Autor para correspondência.

"Departamento de Patologia, Fundação Universidade Federal do Rio Grande (FURG), Rio Grande, RS, Brasil.

"IIFaculdade de Veterinária e Centro de Biotecnologia, UFPel, Pelotas, RS, Brasil.
} 
União Européia a banir, a partir de 2006, o uso de antibióticos como promotores de crescimento nas rações animais (COUNCIL OF EUROPEAN UNION, 2003), comprometendo a eficiência dos sistemas intensivos de produção animal, sustentada em grande parte na utilização de antibióticos como promotores de crescimento ou como preventivos de doenças infecciosas. Uma das doenças mais afetadas por essa medida é a Enterite Necrótica Aviária (ENA) (KALDHUSDAL \& LOVLAND, 2002), um problema freqüente (HOFACRE et al., 1998) e economicamente importante (ENGSTRÖM et al., 2003) em vários países.

A ENA é uma enterotoxemia aguda, que se apresenta em forma clínica ou subclínica (VAN IMMERSEEL et al., 2004), causada por C. perfringens A e C. Caracteriza-se por lesões ulcerativas e necrose confluente da mucosa do intestino delgado e debilidade que se apresenta rapidamente. Afeta principalmente animais jovens, entre duas e cinco semanas de idade, aparecendo subitamente, geralmente associada à imunossupressão, provocando morte rápida com elevada prevalência (SCHOCKEN-ITURRINO \& ISHI, 2000). Embora os casos de ENA não sejam reportados às autoridades sanitárias, é conhecido seu impacto negativo na produção avícola. Estimou-se que nos Estados Unidos o custo dessa doença foi de mais de U\$ 0.05 por animal (VAN DER SLUIS, 2000), podendo provocar prejuízos de até 33\% na produção, principalmente devido ao aumento da conversão alimentar, à redução do peso vivo e ao aumento na condenação de carcaças devido à colangio-hepatite (LOVLAND \& KALDHUSDAL, 2001).

O controle e a prevenção da doença foram baseados durante as últimas décadas na administração de antibióticos na ração, prática comum e necessária para manter e melhorar os índices de produtividade e competitividade.

\section{Etiologia}

A ENA é causada pelo $\boldsymbol{C}$. perfringens, bactéria Gram positiva, anaeróbia, esporulada e toxigênica. $C$. perfringens é classificado em cinco sorotipos em função das toxinas maiores (HATHEWAY, 1990; PETIT et al., 1999). Os sorotipos A e C, produtores de toxina $\alpha$ e $\alpha$ e $\beta$, respectivamente, são considerados os agentes causadores da doença (BABA et al., 1997; LOVLAND \& KALDHUSDAL, 2001), embora a participação de ambos ainda não seja totalmente esclarecida. ENGSTRÖM et al. (2003) detectaram por reação em cadeia da polimerase (PCR) unicamente o gene codificador da toxina $\alpha$ (cpa) em 53 isolados oriundos de intestino e fígado de frangos na Suécia, indicando que todos eles pertenciam ao sorotipo A.
NAUERBY et al. (2003) encontraram o mesmo resultado em 279 isolados de frangos de corte de 25 granjas da Dinamarca, assim como JOHANSSON et al. (2004) em isolados de frangos de corte, galinhas poedeiras e perus, de granjas da Suécia e Dinamarca, utilizando testes bioquímicos e PCR multiplex. HEIKINHEIMO \& KORKEALA (2005) analisaram por PCR multiplex 118 isolados de conteúdo intestinal de frangos de corte e encontraram que todos possuíam o cpa, mas não os genes codificadores das toxinas $ß(c p b), \varepsilon$ (etx), $i(i A)$ e enterotoxina (cpe), que caracterizam os outros sorotipos de $\boldsymbol{C}$. perfringens, indicando que todos os isolados pertenciam ao sorotipo A. Resultados semelhantes foram encontrados por GHOLAMIANDEKHORDI et al. (2006), sugerindo que o agente causador da ENA é o $\boldsymbol{C}$. perfringens sorotipo A.

\section{Epizootiologia}

C. perfringens é um patógeno oportunista (FIORENTIN, 2006) presente no intestino das aves e no ambiente, inclusive na água (SARTORI et al., 2006). Coloniza o animal nos primeiros dias de vida e causa doença principalmente em animais de duas a cinco semanas de idade. Coccidiose tem sido considerada como um importante fator predisponente da ENA(VAN IMMERSEEL et al., 2004). CRAVEN et al. (2001a) encontraram as maiores concentrações da bactéria em fezes de animais com duas e quatro semanas de vida, decaindo na sexta semana e o isolaram, em trabalho subseqüente, de paredes de aviários, ventiladores, comedouros, bebedouros, armadilhas para insetos e botas de operadores, com maior freqüência na primavera e no verão, demonstrando que a estrutura do aviário e seus equipamentos, assim como o incubatório, podem ser fontes de infecção de $\boldsymbol{C}$. perfringens para aves (CRAVEN et al., 2001b).

Fatores ambientais, tais como qualidade da cama, densidade populacional e local de criação, têm grande importância na multiplicação da bactéria e, conseqüentemente, são considerados fatores de risco para a ENA. OMEIRA et al. (2006) avaliaram as características microbiológicas de camas em diferentes sistemas de produção e observaram que poedeiras $\left(0,25 \mathrm{~m}^{2}\right.$ ave $\left.^{-1}\right)$ e frangos de corte $\left(0,1 \mathrm{~m}^{2}\right.$ ave $\left.^{-1}\right)$ em sistema intensivo apresentaram concentrações menores de C. perfringens que criações não-confinadas $\left(0,33 \mathrm{~m}^{2}\right.$ ave $^{-1}$ ), sugerindo que animais em contato com o solo ingerem maior número de esporos da bactéria que animais criados exclusivamente sobre cama em ambiente controlado. McDEVITT et al. (2006) relacionaram o aumento da incidência de ENA à maior densidade animal, devido ao aumento da concentração de esporos 
na cama, associado a sua baixa qualidade (umidade e altos níveis de compostos nitrogenados), além do risco de dispersão por contato direto ou aerossol.

Ingredientes da ração também foram relacionados à doença (DEKICH, 1998). KALDHUSDAL \& SKJERVE (1996) comunicaram que o milho atuou como fator de proteção e cevada e trigo, como fatores de risco para a doença. Observações semelhantes foram feitas por ANNETT et al. (2002), ao comprovarem in vitro que as concentrações da bactéria em meio tioglicolato contendo sobrenadantes não digeridos de cevada e trigo foram maiores que com milho, sugerindo que rações à base de cevada e trigo estimulariam a multiplicação do $\boldsymbol{C}$. perfringens no trato gastrintestinal das aves.

A concentração e as características das proteínas das rações também foram consideradas fatores predisponentes da ENA. DREW et al. (2004) comprovaram aumento da concentração de $\boldsymbol{C}$. perfringens A no íleo e ceco de galinhas quando a concentração de proteína crua de farinha de peixe foi incrementada de 230 para $400 \mathrm{~g} \mathrm{~kg}^{-1}$, não ocorrendo o mesmo em animais alimentados com ração à base de proteína de soja, sugerindo que tanto o nível de proteína crua quanto à fonte protéica utilizados na composição de rações afetam a multiplicação de $\boldsymbol{C}$. perfringens no intestino. DAHIYA et al. (2005) relacionaram os efeitos das concentrações de glicina na ração sobre a população de $\boldsymbol{C}$. perfringens no intestino e as lesões de ENA em frangos de 28 dias de idade desafiados com o microrganismo, constatando que as maiores concentrações da bactéria no ceco foram obtidas com rações contendo de 3,3 a 3,9\%, e que as lesões intestinais variavam de grau zero a quatro nos grupos que receberam 3 e $4 \%$ de glicina.

Insetos também poderiam veicular o agente. DHILLON et al. (2004) reportaram um surto de ENA em galinhas poedeiras de uma granja recém-construída. Eles constataram a presença de moscas no conteúdo do papo dos animais mortos e nos comedouros e isolaram $\boldsymbol{C}$. perfringens de macerados de moscas capturadas nos galpões afetados. Os autores sugerem que o surto foi conseqüência da ingestão do $\boldsymbol{C}$. perfringens presente nas moscas ou suas secreções, considerando esses insetos vetores mecânicos na transmissão da bactéria. VITTORI et al. (2007) isolaram C. perfringens de $100 \%$ de 40 amostras de besouros adultos Alphitobius diaperinus ("Cascudinho") capturados em granjas avícolas industriais de Descalvado e Sertãozinho, SP, Brasil, sugerindo que o Cascudinho pode ser um vetor potencial da veiculação do C. perfringens.

\section{Patogenia}

A ENA é causada pela ação de toxinas produzidas quando, em condições favoráveis, há rápida multiplicação de $\boldsymbol{C}$. perfringens no intestino delgado (SCHOCKEN-ITURRINO \& ISHI, 2000; THOMPSON et al., 2006). As lesões características da ENA são produzidas pela toxina $\alpha$ (WILLIAMS, 2005), a qual vem sendo associada com a doença (TITBALL et al., 1999), sendo considerada o principal fator de patogenicidade da bactéria (DAHIYA et al., 2006; KEYBURN et al., 2006; THOMPSON et al., 2006). A toxina, que destrói a membrana celular de enterócitos devido a sua propriedade de fosfolipase C (STERNE \& BATTY, 1975), é uma metalofosfolipase que possui dois domínios, o C-terminal, que penetra na membrana celular sendo responsável pela fixação da proteína na célula, e o N-terminal, que desempenha a função enzimática propriamente dita e hidrolisa os fosfolipídios das membranas celulares separando as porções polar e apolar, formando di-acil-glicerol e ácido fosfatídico, provocando a lise da membrana celular (SAKURAI et al., 2004).

HOFSHAGEN \& STENWIG (1992) demonstraram que $\boldsymbol{C}$. perfringens isolados de casos de ENA produziram títulos maiores de toxina $\alpha$ que cepas isoladas de animais sadios. Reforçando o conceito de que esta toxina é o principal fator de patogenicidade na ENA, HEIER et al. (2001) observaram que lotes de frangos de corte com altos títulos de anticorpos maternos anti-toxina $\alpha$ apresentaram menores índices de mortalidade que animais com baixos títulos. Entretanto, a participação da toxina $\alpha$ de $\boldsymbol{C}$. perfringens na patogênese foi questionada por KEYBURN et al. (2006), que desafiaram frangos de corte com bactérias cujos genes de toxina $\alpha$ foram deletados, observando que as lesões eram similares às produzidas pela bactéria selvagem.

KULKARNI et al. (2006), procurando identificar antígenos exclusivos a cepas patogênicas de $\boldsymbol{C}$. perfringens envolvidos na patogênese, identificaram anticorpos contra seis proteínas (uma de 190 kDa; Piruvato-ferridoxina-oxidoredutase; Fator G; Perfringolisina O; Gliceraldeido-3-fosfato dehidrogenase e Bifosfato aldolase), em frangos sobreviventes à agressão, sugerindo sua participação na patogênese da ENA, ainda que os soros desses animais também possuíssem anticorpos contra a toxina $\alpha$. THOMPSON et al. (2006), visando também avaliar a participação de outros antígenos na ENA, compararam as taxas de lesões produzidas em frangos por cepas de C. perfringens não-produtoras e uma cepa produtora de toxina $\alpha$, comprovando que não mais de $20 \%$ apresentaram lesões nos primeiros frente a $100 \%$ nos controles positivo, sugerindo que a toxina desempenha um papel importante na patogenia da doença.

Ciência Rural, v.38, n.7, out, 2008. 
Diagnóstico

Em sistemas industriais de produção avícola, a identificação da forma subclínica da doença torna-se difícil por não haver testes adequados, ainda que LOVLAND \& KALDHUSDAL (1999) associaram a hepatite por $\boldsymbol{C}$. perfringens a outras doenças relacionadas à bactéria, sugerindo que o exame de carcaças no abatedouro poderia auxiliar a monitorar a ocorrência da ENA. Na forma clínica, porém, é possível fazer o diagnóstico em função da epidemiologia, da ocorrência da doença com duas a cinco semanas de idade, da intervenção de fatores imunossupressores, tais como alterações bruscas de temperatura, falhas no sistema de arraçoamento, alta densidade populacional, outras doenças, mortalidade alta (de 5 a 15\% do lote), curso rápido sem sinais clínicos e alterações patológicas, tais como hiperemia e lesões ulcerativas na mucosa do intestino delgado e cecos, que se apresentam friáveis, distendidos, com coloração esverdeada escura, fétidos e com gases.

O desenvolvimento de uma técnica imunoenzimática, o ELISA, para quantificar toxina $\alpha$ (HALE \& STILES, 1999), sugeriu que seria possível o diagnóstico das formas clínica e subclínica da ENA. LOVLAND et al. (2003) utilizaram esta técnica para detectar anticorpos anti-toxina $\alpha$ em frangos de corte, constatando a presença de altos títulos em 59\% a 79\% dos animais provenientes de lotes com alta prevalência de lesões hepáticas e intestinais associadas a $\boldsymbol{C}$. perfringens, e só em $27 \%$ dos animais provenientes de lotes com baixa incidência de lesões. Resultados similares foram obtidos por McCOURT et al. (2005) mediante a utilização de ELISA para detecção de células de $\boldsymbol{C}$. perfringens e de toxina $\alpha$ em conteúdo intestinal de frangos. Os testes apresentaram uma sensibilidade entre $10^{2}$ e $10^{6} \mathrm{UFC} \mathrm{mL}^{-1}$ de cepas isoladas de casos clínicos, e de $60 \mathrm{ng} \mathrm{mL} \mathrm{mL}^{-1}$ de toxina. As soroconversões de animais aparentemente saudáveis foram inferiores a 4 , no entanto, as dos afetados foram superiores a 10. Ainda que os testes demonstrassem ser eficientes, o fato de que as amostras utilizadas para detecção do antígeno provinham de conteúdo intestinal, inviabilizando-se assim a amostragem de animais vivos, restringem a aplicação dessa metodologia devido à necessidade de sacrificar uma amostra significativa de animais do lote, o que é inaceitável na avicultura industrial.

Controle

O controle da ENA foi baseado, nas últimas décadas, na administração de antibióticos na ração (ENGBERG et al., 2000; BRENNAN et al., 2001; KNARREBORG et al., 2002; BRENNAN et al., 2003). A interdição de seu uso como promotores de crescimento, decretada pela União Européia (COUNCIL OF THE EUROPEAN UNION, 2003), lançou o desafio de criar novas estratégias para controlar a doença. Surgiu então o interesse em avaliar métodos alternativos, tais como o uso de prebióticos (TAKEDA et al., 1995; SILVA \& NORNBERG, 2003; MCREYNOLDS et al., 2007), enzimas (JACKSON et al., 2003; ZANG et al., 2006), alimentos funcionais (MITSCH et al., 2004), probióticos (LA RAGIONE et al., 2004; BARBOSA et al., 2005; KIZERWETTER-SWIDA \& BINEK, 2005) e vacinas. Tanto o setor industrial quanto o acadêmico tem demonstrado grande interesse no uso dos dois últimos para o controle da ENA.

\section{Probióticos}

Probióticos são suplementos alimentares compostos de microrganismos vivos que beneficiam a saúde do hospedeiro por meio do equilíbrio da microbiota intestinal (FULLER, 1989; KAUR et al., 2002). Sua aplicação na indústria avícola vem sendo amplamente estudada nos últimos anos, tanto para o controle de doenças quanto por seu efeito na eficiência alimentar (GIL de los SANTOS \& GIL-TURNES, 2005).

HOFACRE et al. (1998) observaram que o produto Aviguard ${ }^{\circledR}$, constituído por microbiota polibacteriana de aves, reduziu a incidência de ENA em frangos de corte. LA RAGIONE \& WOODWARD (2003) comprovaram que a administração de esporos viáveis de Bacillus subtilis a aves livres de patógenos específicos desafiadas com $\boldsymbol{C}$. perfringens reduziu o numero de patógenos no baço, no duodeno, no cólon e no ceco e relataram resultados similares com um probiótico de Lactobacillus johnsonii (LA RAGIONE et al., 2004). TEO \& TAN (2005), por sua vez, demonstraram que B. subtilis inibiu o crescimento de $\boldsymbol{C}$. perfringens em cultivo associado. HAGHIGHI et al. (2006) demonstraram que um probiótico comercial contendo Lactobacillus acidophilus, Bifidobacterium bifidum, e Streptococcus faecalis estimulou a produção de IgA anti-toxina $\alpha$ de C. perfringens no intestino de pintos não-vacinados.

\section{Vacinas}

Aves que sobreviveram à ENA ficaram imunes, sugerindo que a imunidade pode controlar a doença (PRESCOTT, 2000). LOVLAND et al. (2004) demonstraram, pela primeira vez, que progênies de matrizes imunizadas com um toxóide de $\boldsymbol{C}$. perfringens A apresentaram títulos de antitoxina significativamente superiores aos controles, assim como menor porcentagem de animais com lesões de ENA. A produção industrial de toxóides de $\boldsymbol{C}$. perfringens, 
porém, é um processo laborioso. A clonagem do gene da toxina $\alpha$ em Escherichia coli (LESLIE et al., 1989) e a produção de vacinas contendo toxina $\alpha$ recombinante (WILLIAMSON \& TITBALL, 1993; SCHOEPE et al., 2001; STEVENS et al., 2004; SCHOEPE, 2006) abriram uma nova perspectiva de produção industrial de antígenos de Clostridium.

KULKARNI et al. (2007) vacinaram animais com a toxina $\alpha$ nativa e com proteínas recombinantes secretadas de $\boldsymbol{C}$. perfringens (piruvato-ferridoxinaoxidoredutase; gliceraldeido-3-fosfato dehidrogenase; frutose 1,6-bifosfato aldolase e uma proteína hipotética), comprovando que todas as vacinas apresentaram efeito protetor frente a um desafio moderado, e que frente a um desafio severo a toxina $\alpha$ apresentou o melhor resultado, reafirmando sua importância tanto na patogenia do $\boldsymbol{C}$. perfringens, quanto na imunidade a esse patógeno. Entretanto, ao tentarem produzir a toxina $\alpha$ recombinante, concluíram que a mesma pareceria ser tóxica para $\boldsymbol{E}$. coli. Nosso grupo, porém, logrou produzir toxina $\alpha$ recombinante em $\boldsymbol{E}$. coli que protegeu camundongos frente ao desafio com mais de $10 \mathrm{DL}_{50}$ de toxina nativa e imunizou pintos vacinados aos sete dias de idade (GIL de los SANTOS, 2007).

\section{CONSIDERAÇÕES FINAIS}

O controle da ENA é considerado um dos maiores desafios para a avicultura industrial. Entre as alternativas para consegui-lo, poderão ser utilizados probióticos, que mantém o equilíbrio da microbiota do trato gastrintestinal de aves, previnem infecções, reduzem condenações de carcaças e a mortalidade, melhoram a conversão alimentar, o ganho de peso e a qualidade das carcaças, conservando os índices de produtividade alcançados com a utilização de antimicrobianos e vacinas, especialmente as recombinantes, de produção mais simples e controlável que as tradicionais. Nutrição adequada e controle de fatores imunossupressores, conjuntamente com um eficiente programa de biosseguridade, são essenciais para o êxito do controle da ENA.

\section{REFERÊNCIAS}

ANNETT, C.B. et al. Necrotic enteritis: effect of barley, wheat and corn diets on proliferation of Clostridium perfringens type A. Avian Pathology, v.31, p.599-602, 2002.

BABA, E. et al. Clostridial population and the intestinal lesions in chickens infected with Clostridium perfringens and Eimeria necatrix. Veterinary Microbiology, v.54, p.301-308, 1997.
BARBOSA, T.M. et al. Screening for Bacillus isolates in the broiler gastrointestinal tract. Applied and Environmental Microbiology, v.71, p.968-978, 2005.

BRENNAN, J. et al. Efficacy of in-feed tylosin phosphate for the treatment of Necrotic Enteritis in broiler chickens. Poultry Science, v.80, p.1451-1454, 2001.

BRENNAN, J. et al. The efficacy of bacitracin methylene disalicylate when fed in combination with narasin in the management of Necrotic Enteritis in broiler chickens. Poultry Science, v.82, p.360-363, 2003.

COUNCIL OF EUROPEAN UNION. Council Regulation on the authorization of the additive avilamycin in feedingstuffs. 2003. Capturado em 17 out. 2003. Online. Disponível na Internet: http://register.consilium.eu.int/pdf/en/ 03/st06/st06120en03.pdf.

CRAVEN, S.E. et al. Incidence of Clostridium perfringens in broiler chickens and their environment during production and processing. Avian Diseases, v.45, n.4, p.887-896, 2001a.

CRAVEN, S.E. et al. Prevalence of Clostridium perfringens in commercial broiler hatcheries. Avian Diseases, v.45, n.4, p.1050-1053, 2001b.

DAHIYA, J.P. et al. Dietary glycine concentration affects intestinal Clostridium perfringens and Lactobacilli populations in broiler chickens. Poultry Science, v.84, p.1875-1885, 2005.

DAHIYA, J.P. et al. Potential strategies for controlling necrotic enteritis in broiler chickens in post-antibiotic era. Animal Feed Science and Technology, v.129, p.60-88, 2006.

DEKICH, M.A. Broiler industry strategies for control of respiratory and enteric diseases. Poultry Science, v.77, p.1176-1180, 1998.

DHILLON, A.S. et al. High mortality in egg layers as a result of necrotic enteritis. Avian Diseases, v.48, n.3, p.675-680, 2004 .

DREW, M.D. et al. Effects of dietary protein source and level on intestinal populations of Clostridium perfringens in broiler chickens. Poultry Science, v.83, n.3, p.414-420, 2004.

ENGBERG, R.M. et al. Effect of zinc bacitracin and salinomycin on intestinal microflora and performance of broilers. Poultry Science, v.79, p.1311-1319, 2000.

ENGSTRÖM, B.E. et al. Molecular typing of isolates of Clostridium perfringens from healthy and diseased poultry. Veterinary Microbiology, v.94, n.3, p.225-235, 2003.

FIORENTIN, L. Aspectos bacteriológicos da reutilização da cama de aviário. In: SEMINÁRIO INTERNACIONAL DE AVES E SUÍNOS - AVESUI, 5., 2005, Florianópolis, SC. Anais... Itu, SP: GESSULLI AGRIBUSINESS, 2006, v.1, p.113-122.

FULleR, R. Probiotics in man and animals. Applied Bacteriology, v.66, n.5, p.365-378, 1989.

GHOLAMIANDEKHORDI, A.R. et al. Molecular and phenotypical characterization of Clostridium perfringens 
isolates from poultry flocks with different disease status. Veterinary Microbiology, v.113, n.1-2, p.143-152, 2006.

GIL de los SANTOS, J.R. Construção e avaliação de vacinas de toxina $\alpha$ recombinante de Clostridium perfringens $A$. 2007. 82f. Tese (Doutorado em Biotecnologia Agrícola) Programa de Pós-graduação em Biotecnologia Agrícola, Universidade Federal de Pelotas.

GIL de los SANTOS, J.R.; GIL-TURNES, C. Probióticos em avicultura. Ciência Rural, v.35, n.3, p.741-747, 2005.

HAGHIGHI, H.R. et al. Probiotics stimulate production of natural antibodies in chickens. Clinical and Vaccine Immunology, v.13, n.9, p.975-980, 2006.

HALE, M.L.; STILES, B.G. Detection of Clostridium perfringens alpha toxin using a capture antibody ELISA. Toxicon, v.37, n.3, p.471-484, 1999.

HATHEWAY, C.H. Toxigenic Clostridia. Clinical Microbiology Reviews, v.3, n.1, p.66-98, 1990.

HEIER, B.T. et al. A field study of naturally occurring specific antibodies against Clostridium perfringens alpha toxin in Norwegian broiler flocks. Avian Diseases, v.45, n.3, p.724732, 2001.

HEIKINHEIMO, A.; KORKEALA, H. Multiplex PCR assay for toxinotyping Clostridium perfringens isolates obtained from Finnish broiler chickens. Letters in Applied Microbiology, v.40, p.407-411, 2005

HOFACRE, C.L. et al. Use of Aviguard and other intestinal bioproducts in experimental Clostridium perfringens-associated Necrotizing Enteritis in broiler chickens. Avian Diseases, v.42, p.579-584, 1998

HOFSHAGEN, M.; STENWIG, H. Toxin production by Clostridium perfringens isolated from broiler chickens and capercaillies (Tetrao urogallus) with and without Necrotizing Enteritis. Avian Diseases, v.36, n.4, p.837-843, 1992.

IBGE - Instituto Brasileiro de Geografia e Estatística. 2007. Capturado em 01 set. 2007. Online. Disponível na Internet: http://www.ibge.gov.br

JACKSON, M.E. et al. Beneficial effect of beta-mannanase feed enzyme on performance of chicks challenged with Eimeria sp. and Clostridium perfringens. Avian Diseases, v.47, n.3, p.759-63, 2003.

JOHANSSON, A. et al. Antimicrobial susceptibility of Swedish, Norwegian and Danish isolates of Clostridium perfringens from poultry, and distribution of tetracycline resistance genes. Veterinary Microbiology, v.99, p.251-257, 2004.

KALDHUSDAL, M.; LOVLAND, A. Clostridial necrotic enteritis and cholangiohepatitis - 'Biography'. In: THE ELANCO GLOBAL ENTERITIS SYMPOSIUM, 2002, Cambridge, UK. Anais... p. G3-G13. Capturado em $8 \mathrm{dez}$. 2003. Online. Disponível na Internet: http://www.poultryhealth.com/fora/inthelth/kaldhusdal02.pdf

KALDHUSDAL, M.; SKJERVE, E. Association between cereal contents in the diet and incidence of necrotic enteritis in broiler chickens in Norway. Preventive Veterinary Medicine, v.28, p.1-16, 1996.

KAUR, I.P. et al. Probiotics: potential pharmaceutical applications. European Journal of Pharmaceutical Sciences, v.15, p.1-9, 2002.

KEYBURN, A.L. et al. Alpha-toxin of Clostridium perfringens is not an essential virulence factor in Necrotic Enteritis in chickens. Infection and Immunity, v.74, n.11, p.6496-6500, 2006.

KIZERWETTER-SWIDA, M.; BINEK, M. Selection of potentially probiotic Lactobacillus strains towards their inhibitory activity against poultry enteropathogenic bacteria. Polish Journal of Microbiology, v.54, n.4, p.287-294, 2005.

KNARREBORG, A. et al. Effects of dietary fat source and subtherapeutic levels of antibiotic on the bacterial community in the ileum of broiler chickens at various ages. Applied and Environmental Microbiology, v.68, n.12, p.5918-5924, 2002.

KULKARNI, R.R. et al. Clostridium perfringens antigens recognized by broiler chickens immune to necrotic enteritis. Clinical and Vaccine Immunology, v.13, n.12, p.13581362, 2006

KULKARNI, R.R. et al. Immunization of broiler chickens against Clostridium perfringens-induced necrotic enteritis. Clinical and Vaccine Immunology. In press, 2007. doi:10.1128/CVI.00162. Capturado em 8 ago. 2007. Online. Disponível na Internet: http://cvi.asm.org/cgi/reprint/ CVI.00162-07v1.pdf

LA RAGIONE, R.M.; WOODWARD, M.J. Competitive exclusion by Bacillus subtilis spores of Salmonella enterica serotype Enteritidis and Clostridium perfringens in young chickens. Veterinary Microbiology, v.94, n.3, p.245-256, 2003.

LA RAGIONE, R.M. et al. In vivo characterization of Lactobacillus johnsonii FI9785 for use as a defined competitive exclusion agent against bacterial pathogens in poultry. Letters in Applied Microbiology, v.38, p.197-205, 2004.

LESLIE, D. et al. Phospholipase C and haemolytic activities of Clostridium perfringens alpha-toxin cloned in Escherichia coli: sequence and homology with a Bacillus cereus phospholipase C. Molecular Microbiology, v.3, n.3, p.383392, 1989.

LOVLAND, A.; KALDHUSDAL, M. Liver lesions seen at slaughter as an indicator of Necrotic enteritis in broiler flocks. FEMS Immunology and Medical Microbiology, v.24, n.3, p.345-351, 1999 .

LOVLAND, A.; KALDHUSDAL, M. Severely impaired production performance in broiler flocks with high incidence of Clostridium perfringens-associated hepatitis. Avian Pathology, v.30, p.73-81, 2001

LOVLAND, A. et al. Diagnosing Clostridium perfringensassociated necrotic enteritis in broiler flocks by an immunoglobulin G anti-alpha-toxin enzyme-linked immunosorbent assay. Avian Pathology, v.32, n.5, p.527534, 2003. 
LOVLAND, A. et al. Maternal vaccination against subclinical necrotic enteritis in broilers. Avian Pathology, v.33, n.1, p.83-92, 2004.

MARTEL, A. et al. Susceptibility of Clostridium perfringens strains from broiler chickens to antibiotics and anticoccidials. Avian Pathology, v.33, n.1, p.3-7, 2004.

McCOURT, M.T. et al. Sandwich ELISA detection of Clostridium perfringens cells and $\alpha$-toxin from field cases of necrotic enteritis of poultry. Veterinary Microbiology, v.106, n.3-4, p.259-264, 2005.

McDEVITT, R.M. et al. Necrotic enteritis; a continuing challenge for the poultry industry. World's Poultry Science Journal, v.62, n.2, p.221-247, 2006.

McREYNOLDS, J.L. et al. Dietary lactose and its effect on the disease condition of Necrotic Enteritis. Poultry Science, v.86, n.8, p.1656-1661, 2007.

MITSCH, P. et al. The effect of two different blends of essential oil components on the proliferation of Clostridium perfringens in the intestines of broiler chickens. Poultry Science, v.83, p.669-675, 2004.

NAUERBY, B. et al. Analysis by pulsed-field gel electrophoresis of the genetic diversity among Clostridium perfringens isolates from chickens. Veterinary Microbiology, v.94, n.3, p.257266, 2003.

OMEIRA, N. et al. Microbiological and chemical properties of litter from different chicken types and production systems. Science of the Total Environment, v.367, p.156-162, 2006.

PETIT, L. et al. Clostridium perfringens: toxinotype and genotype. Trends in Microbiology, v.7, n.3, p.104-110, 1999.

PRESCOTT, J. Vaccine-based control of Necrotic Enteritis of broiler chickens. Ministry of Agriculture and Food, Ottawa, Canada, 2000. Capturado em 23 jan. 2004. Online. Disponível na Internet: http://www.omaf.gov.on.ca/english/livestock/ poultry/facts/necrente.htm

SAKURAI, J. et al. Clostridium perfringens alpha-toxin: Characterization and mode of action. Journal of Biochemistry, v.136, n.5, p.569-574, 2004.

SARTORI, D.P. et al. Evaluation of acid phosphatase as a confirmation test for Clostridium perfringens isolated from water. Letters in Applied Microbiology, v.42, n.4, p.418424, 2006.

SCHOCKEN-ITURRINO, R.P; ISHI, M. Clostridioses em aves. In: BERCHIERI Jr, A.; MACARI, M. Doenças das aves. Campinas: Facta, 2000. Cap.4.6, p.242-243.

SCHOEPE, H. et al. Naturally occurring Clostridium perfringens nontoxic alpha-toxin variant as a potential vaccine candidate against alpha-toxin-associated diseases. Infection and Immunity, v.69, n.11, p.7194-7196, 2001.
SCHOEPE, H. et al. Immunization with an alphatoxin variant 121A/91-R212H protects mice against Clostridium perfringens alphatoxin. Anaerobe, v.12, p.44-48, 2006.

SILVA, L.P.; NORNBERG, J.L. Prebióticos na nutrição de não ruminantes. Ciência Rural, v.33, n.5, p.983-990, 2003.

SINGH, R.V. et al. Studies on occurrence and characterization of Clostridium perfringens from select meat. Journal of Food Safety, v.25, p.146-156, 2005.

STERnE, M.; BATTY, I. Pathogenic clostridia. London: Butterworths, 1975. 144p.

STEVENS, D.L. et al. Immunization with the C-domain of $\alpha$ toxin prevents lethal infection, localizes tissue injury, and promotes host response to challenge with Clostridium perfringens. Journal of Infectious Diseases, v.190, p.767773, 2004.

TAKEDA, T. et al. The effects of dietary lactose and rye on cecal colonization of Clostridium perfringens in chicks. Avian Diseases, v.39, n.2, p.375-381, 1995.

TEO, A.Y.L.; TAN, H.M. Inhibition of Clostridium perfringens by a novel strain of Bacillus subtilis isolated from the gastrointestinal tracts of healthy chickens. Applied and Environmental Microbiology, v.71, n.8, p.4185-4190, 2005.

THOMPSON, D.R. et al. Live attenuated vaccine-based control of necrotic enteritis of broiler chickens. Veterinary Microbiology, v.113, n.1-2, p.25-34, 2006.

TITBALL, R.W. et al. The Clostridium perfringens $\alpha$-toxin. Anaerobe, v.5, p.51-64, 1999.

VAN DER SLUIS, W. Clostridial enteritis is an often underestimated problem. World Poultry, v.16, n.7, p.42-43, 2000 .

VAN IMMERSEEL, F. et al. Clostridium perfringens in poultry: an emerging threat for animal and public health. Avian Pathology, v.33, n.6, p.537-549, 2004.

VITTORI, J. et al. Alphitobius diaperinus como veiculador de Clostridium perfringens em granjas avícolas do interior paulista - Brasil. Ciência Rural, v.37, n.3, p.894-896, 2007.

WILLIAMS, R.B. Intercurrent coccidiosis and necrotic enteritis of chickens: rational, integrated disease management by maintenance of gut integrity. Avian Pathology, v.34, n.3, p.159-180, 2005.

WILLIAMSON, E.D.; TITBALL, R.W. A genetically engineered vaccine against the alpha-toxin of Clostridium perfringens protects mice against experimental gas gangrene. Vaccine, v.11, n.12, p.1253-1258, 1993.

ZANG, G. et al. In vitro inhibitory effect of hen egg white lysozyme on Clostridium perfringens type A associated with broiler necrotic enteritis and its $\alpha$-toxin production. Letters in Applied Microbiology, v.42, n.2, p.138-143, 2006. 